\title{
Buchbesprechungen - Books Review - Livres Nouveaux
}

W. Teschendorf: Lehrbuch der röntgenologischen Differentialdiagnostik. Bd. II. Erkrankungen der Bauchorgane. Georg Thie $\alpha$ e, Stuttgart 1950. 2. Auflage, 608 Seiten. DM 72.-.

Die Röntgenuntersuchung ist im Laufe der letzten Jahrzehnte die wichtigste

Untersuchungsmethode bei Erkrankungen der Bauchorgane geworden. Das wird so recht deutlich, wenn man die Fülle von Befunden übersieht, welche im vorlie-genden Werk zu einer systematischen Übersieht zusammengefaßt und durch 1081 vorzügliche Abbildungen illustriert wird.

Der 1. Abschnitt ist den akuten Baucherkrankungen gewidmet; dann folgen in 6 Kapiteln die Röntgenologie der Verdauungsorgane. Die Darstellung ist im allgemeinen topographisch, indem die einzelnen Organe oder Organteile und ihre Beziehung zur Umgebung systematisch abgehandelt werden. Alle differential-diagnostischen Uberlegungen werden klar herausgearbeitet. Ein einziger Wunsch sei hier geäußert, nämlich der, daß in einer weiteren Auflage bei der Frühdiagnose des Magenkrebses die Ergebnisse der Untersuchungen Gutmanns ihren gebühren-den Platz finden mögen.

Der letzte Abschnitt umfaßt die Röntgenuntersuchung der Niere und der ab-leitenden Harnwege.

Das Buch bietet dem Anfanger eine vorzügliche Einführung und bleibt dem

Erfahrenen ein unentbehrliches Nachschlagewerk. Kapp.

Hess, W.: Chirurgie des Pancreas. Benno Schwabe, Bale 1950, 156 pp.

Dans sa preface, le Prof. Schürch annonce que ce livre embrasse toute la litté-

Buchbesprechungen - Books Review - Livres Nouveaux 327

rature de 1937 à 1949 sur le pancreas. H. tient cette promesse; mais sa mono-graphie est beaucoup mieux qu'une «revue d'ensemble», car le millier de publications indexêes dans la bibliographie est passé au crible d'une critique constructive, basée sur une vaste experience personnelle. Cet effort de synthèse mérite d'etre souligné; $\Gamma$ A. réussit à condenser en 130 pages une dizaine de courts chapitres traitant successivement des pancréatites aiguë, cbronique et litbiasique, des affections congênitales et traumatiques, de kystes, fistules et tumeurs de la glande (y compris les insulomes). II a même la place de consacrer un paragraphe aux relations du pancreas avec les organes voisins ou distants (voies biliaires, thyroïde, système osseux, diabète). La partie la plus moderne et originale est celle réservée à la pancréatectomie; les anastomoses multiples nécessitêes par cette grave intervention sont illustrées par 2 pages de schémas particulièrement clairs; la technique opératoire représentée par 12 remarquables photographies exposant ses temps successifs.

Si ce livre, écrit par un chirurgien, est destine avant tout aux chirurgiens, en leur permettant de se familiariser, par une soiree de lecture, avec ces problèmes complexes, il n'en intéressera pas moins les internistes qui ne peuvent rester indifférents à une question en pleine evolution. M. Demole.

Ivy, A. C, Grossman, M. I. et W. H. Bachrach: Peptic ulcer. Blakiston edit. Philadelphia 1950. Voici un gros volume - plus de 1150 pages - que tout spécialiste regrettera de n'avoir pas parcouru, "a must book". II a été construit autour d'une these de doctorat (celle de B.) sur 
Tulcère experimental, et les A. sont d'ailleurs avant tout des physiologistes; aussi ne s'étonne-ton pas de les voir consacrer les deux tiers du volume à la pathogenic Mais comme il est dit dans la preface, après sa lecture, la classique question "where do we go from here?" se remplace par Tindication "where we should go from here", c'est-à-dire qu' on en tire une lígne de conduíte, basée sur une meilleure comprehension de la maladie.

La premiere partie est fondamentale: definition de $\Gamma$ ulcère; physiologie de la secretion gastrique; resistance de Гestomac à la digestion; guérison des ulcères aigus et chroniques.

La seconde partie, sous prétexte de pathogénie, envisage en réalité toute la patho-logíe de

Tulcère en une série de chapitres: histo-physiologie; role des secretions gastro-duodénales, du système nerveux; production expérimentale de Гulcère «peptique». Puis, ranges sous le même sous-titre, des considerations sur la frequence, clinique ou autopsique; sur la localisation; sur les complications: perforation et hémorragie; sur Гaspect constitutionnel et $\Gamma$ étiologie psychosomatique; sur le mécanisme de la douleur etc.

Les problèmes du diagnostic $-\mathrm{y}$ compris celui de $\Gamma$ ulcère peptique anastomotique, des complications et une discussion de la dégénérescence maligne - sont traités en moins de 100 pages, et sans un seul cliche radiologique! Et $\Gamma$ on est stupéfait de ne pas trouver, parmi les 5000 cotes bibliographiques, des noms comme celui de Gut-mann! Ce livre n'est done pas destine au praticien, mais au clinicien et au chercheur, curieux de pénétrer plus avant dans la connaissance d'une affection où subsistent tant d'inconnues essentielles, malgré sa frequence.

La quatrième partie (thérapeutique) ne se contente pas d'une enumeration des moyens de traitement, mais discute leur mécanisme d'action, leur efficacité relative,

328 Buchbesprechungen - Books Review - Livres Nouveaux

Гutilité du repos; les facteurs de rechute et les elements du pronostic et de la durée des guérisons; les diverses techniques chirurgicales et leurs indications.

En parlant de parcourir cette magnifique monographie, nous ne voulons pas dire qu'elle ne mérite pas une lecture attentive; au contraire, on ne se contentera pas de lire ce texte dense et precis; il inspire la reflexion, la discussion intérieure, doit être lu par fragments successifs. Mais chaque chapitre est si clairement divisé, qu'on peut fort bien tourner rapidement plusieurs pages, pour s'arrêter plus longuement sur certains paragraphes qui forment un tout par eux-mêmes; ils sont terminés chacun par un resume et par une abondante bibliographie, de sorte que Touvrage d'Juy et ses collaborateurs représente par excellence un de ces livres de fond, auquel on reviendra souvent. M. Demole.

Bücker, J.: Gastritis, Ulkus und Karzinom. Röntgenstudie unter Berücksichtigung formalgenetischer Beziehungen. Georg Thieme, Stuttgart, 1950, 89 S., 82 Abb. 11.50 DM. Die Röntgenuntersucbung der drei wichtigsten Magenkrankheiten ist noch nicht abgeschlossen. Der Autor zeigt in der vorliegenden Studie, was sie leisten kann, wenn man das Bild von der pathologisch-anatomischen Veränderung der Magenwand her erklärt. Er bevorzugt die Methode der dünnen Schicht mit gezielten Aufnahmen und Verfolgen jeder einzelnen Falte beim Durchleuchten. Bei der Gastritis werden eindrücklich die plastische Pangastritis und die verschiedenen Formen der Antrumgastritis (plastische, stenosierende, hypertrophisch beetförmige, erosive) analysiert. Häufig wird dem Röntgenbild das anatomische Präparat gegenübergestellt. Besonderes Interesse verdient die Darstellung der Gastritis granularis. Im kurzen Kapitel über das Magengeschwür wird besonders auf die Veränderungen der Magenwand eingegangen, welche die Größe des Nischenschattens bedingen. Das 
Röntgenbild des Magenkrebses wird klar herausgearbeitet, und zahlreiche kleine Karzinome sind dargestellt. Allerdings fehlen die Friihformen im engeren Sinne, wie sie von Gutmann, Guy Albot und anderen beschrieben wurden. Auswahl und Wiedergabe der Röntgenbilder sind musterhaft. Kapp Kuhlmann, F,: Der Dünndarm im Röntgenbild. Urban \& Schwarzenberg, München, Berlin, Wien, 1951. 110 S., 80 Abb., DM 11.80.

Die Diagnose der Dünndarmerkrankungen ist schwierig, und die Röntgenunter-suchung, welche die wichtigste Methode auf diesem Gebiet ist, arbeitet noch nicht mit Sicherheit. Es ist daher erfreulich, daß der Autor seine große Erfahrung zu-sammenfassend darstellt. Nach einer kurzen Besprechung der Untersuchungstech-nik und einigen physiologischen Bemerkungen werden die röntgenologisch erfaß-baren Dünndarmstörungen eingehend abgehandelt. Bei der Analyse von Lage, Motilität, Tonus, Sekretion, Gasbildungen und Schleimhautbeschaffenheit werden alle Erscheinungen festgelegt, welche durch pathologische Prozesse im Dünndarm hervorgerufen werden. Im Hauptteil der Monographie werden die speziellen Krank-heitsbilder abgehandelt: akute Enteritis, Darmbrand, chronische Enteritis, Vit-aminmangelzustände, regionäre Jejunitis und Ileitis, Allergie, Darmtuberkulose, Bauchfellerkrankungen und ihre Beziehungen zum Dünndarm, Ileus, Tumoren, Parasiten. Die Ausstattung des Buches ist gut. Ein Literaturverzeichnis fehlt.

Kapp 